\title{
CAPACIDADE VITAL E TEMPOS MÁXIMOS DE FONAÇÃO DE /E/ ÁFONO E DE /S/ EM MULHERES ADULTAS
}

\author{
Vital capacity and maximum phonation times \\ of voiceless $/ \mathrm{e} /$ and $/ \mathrm{s} /$ in adult women
}

\author{
Shanna Lara Miglioranzi ${ }^{(1)}$, Carla Aparecida Cielo(2), Márcia do Amaral Siqueira(3)
}

\begin{abstract}
RESUMO
Objetivo: verificar a capacidade vital (CV) e os valores de tempo máximo de fonação (TMF) do /e/ áfono (representado por /è/) e do /s/ de mulheres adultas, estabelecendo o perfil da amostra e comparando-o com o padrão de normalidade proposto. Método: coleta do maior valor de três medidas de $\mathrm{CV}$, de TMF/è/ e de TMF/s/; e da estatura auto-referida de 48 mulheres entre 18 e 44 anos de idade, normais de acordo com avaliações otorrinolaringológica, miofuncional, vocal, respiratória e auditiva. Aplicou-se análise estatística descritiva, teste de normalidade de Shapiro-Wilk, e cálculo do coeficiente de variação; adotou-se o nível de significância de 5\%. Resultados: valores médios de CV de $3.206 \mathrm{ml}$, de TMF/s/ de 17,49s e de estatura de 1,65m, com distribuição normal; TMF/è/ sem distribuição normal e média de 10,43s significantemente menor do que os valores da literatura $(\mathrm{P}<0.001)$. No entanto, o TMF/è/ apresentou valores de média e mediana bastante próximos $(10,43 \mathrm{~s}$ e 10,25s) e coeficiente de variação muito similar ao TMF/s/. Conclusão: para o grupo de analisado, encontraram-se valores médios de $\mathrm{CV}$ e de TMF/s/ compatíveis com os referidos pela literatura. Os valores médios de TMF/è/ se apresentaram abaixo do proposto pela única referência teórica existente, evidenciando a necessidade de mais pesquisas como esta - que investiguem em campo a medida de TMF/è/ - a fim de estabelecer a faixa normalidade conforme o sexo e obter mais dados científicos sobre o que parece ser a medida mais indicada para a avaliação isolada do controle respiratório durante a emissão.
\end{abstract}

DESCRITORES: Voz; Pregas Vocais; Capacidade Vital; Distúrbios da Voz

\section{INTRODUÇÃO}

Os dados aerodinâmicos podem ser usados para auxiliar o diagnóstico diferencial entre alterações

(1) Fonoaudióloga; Consultório particular, Santa Maria, RS; Mestre em Distúrbios da Comunicação Humana pela Universidade Federal de Santa Maria - UFSM; Santa Maria, RS, Brasil.

(2) Fonoaudióloga; Professora Adjunta dos cursos de Graduação em Fonoaudiologia e Pós-Graduação em Distúrbios da Comunicação Humana do Departamento de Fonoaudiologia da Universidade Federal de Santa Maria - UFSM - Santa Maria, RS; Doutora em Linguística Aplicada pela Pontifícia Universidade Católica do Rio Grande do Sul PUC/RS - Santa Maria, RS, Brasil.

(3) Fonoaudióloga do Centro de Referência em Saúde do Trabalhador-Centro/Santa Maria, RS; Mestre em Distúrbios da Comunicação Humana pela Universidade Federal de Santa Maria - UFSM - Santa Maria, RS, Brasil.

Conflito de interesses: inexistente laríngeas, de controle respiratório e condições pulmonares, ou seja, em casos de problemas de voz, por meio dessas medidas, pode-se tentar inferir se existe contribuição laríngea ou se são decorrentes de falta de controle respiratório ou das condições do pulmão ${ }^{1-9}$.

$\mathrm{Na}$ avaliação das medidas respiratórias, a medida da capacidade vital $(\mathrm{CV})$ é comumente utilizada. A CV é a quantidade máxima de ar que se pode expirar dos pulmões, em seguida a uma inspiração máxima ${ }^{2-4,8,10}$. Representa 0 maior volume de ar mobilizado ${ }^{8}$. A CV varia enormemente entre indivíduos ${ }^{8}$, de acordo com a altura ${ }^{3,4}$, raça, sexo, saúde, hábitos como fumo e a prática de esportes $^{4,8,11}$, e com a faixa etária ${ }^{7,10,12}$. Sugere-se, como mínimo, o valor de $2.100 \mathrm{ml}$ para mulheres e $2.200 \mathrm{ml}$ para homens $\mathrm{s}^{2,13,14}$.

A medida dos tempos máximos de fonação (TMF) avalia a eficiência da coordenação laríngea 
e respiratória, fornecendo dados objetivos sobre a dinâmica vocal, bem como dados sobre a qualidade vocal. Os TMF registram o máximo intervalo de tempo que o indivíduo é capaz de sustentar um som em uma expiração prolongada. Constituem-se em um instrumento fácil, prático e efetivo para a avaliação, diagnóstico e evolução do paciente em fonoterapia. Essa medida inclui as vogais e fricativas surdas e sonoras ${ }^{1,3,4,6-8,15,16}$.

Tratando-se de uma medida quantitativa, permite, ainda, a padronização e normatização de seus valores, acrescentando maior fidedignidade ao possibilitar a comparação entre as diferentes populações.

Dentre as medidas dos TMF, destaca-se, neste estudo, a medida dos TMF dos fonemas $/ \mathrm{s} /{ }^{3-6,8,16} \mathrm{e}$ de /e/ áfono (representado por /è//), que não se utilizam da fonte glótica, evidenciando, assim, o controle respiratório durante a emissão.

O fonema /s/, apesar de não utilizar a vibração das pregas vocais, apresenta constrição no nível articulatório alveolar, influenciando o controle da saída do ar expiratório4-6,8,16.

A normalidade para o TMF de /s/ de adultos, na literatura revisada, está em torno de 15 a 25 segundos. Valores inferiores indicam comprometimento do suporte respiratório $0^{3,4,8,13,16}$.

Afirma-se que a medida de /è/ indica de forma mais fidedigna o controle da saída do ar através de seu suporte respiratório, pois a sustentação de seu tempo de emissão depende quase que exclusivamente do controle respiratório ${ }^{4,8}$. As fricativas, ao contrário, estão submetidas à barreira ou bloquieo articulatório ${ }^{4,6,8,16}$.

Sugerem-se medidas de normalidade em torno de 16 a 18 segundos para o /è/, evidenciando deficiências no mecanismo respiratório para tempos de emissão inferiores a 16 segundos ${ }^{4,8}$.

Os valores de TMF estão comumente associados à efetividade da adução glótica e aos problemas de controle respiratório ${ }^{4,9}$, isto é, quanto menor o TMF, maior o distúrbio de controle glótico e ou respiratório.

Verificou-se escassez de estudos sobre tais medidas, sendo que há apenas uma referência teórica, sem pesquisa de campo, publicada em literatura nacional sobre o TMF/è/ e que não faz distinção da faixa de normalidade entre os sexos ${ }^{4}$. E outra pesquisa que utiliza o TMF/è/ como uma medida de coleta de dados dos sujeitos ${ }^{8}$.

Desta forma, o presente estudo se propõe a verificar a CV e os valores de TMF de /è/ e de /s/ de mulheres adultas, estabelecendo o perfil da amostra e comparando-o com o padrão de normalidade proposto.

\section{MÉTODO}

Esta pesquisa constitui um estudo quantitativo, transversal e exploratório, por meio de levantamento de dados em banco de dados da clínicaescola da instituição de origem (primeira etapa, de caráter retrospectivo) e por meio de coleta em campo (segunda etapa, de caráter contemporâneo).

A população-alvo consistiu de mulheres no último ano da adolescência e na fase adulta, sendo foram considerados os seguintes critérios de inclusão:

- ser do sexo feminino, pela facilidade de captação de voluntários para pesquisa ${ }^{17}$;

- estar no último ano da adolescência (18 anos) ou na fase adulta (19 a 44 anos de idade) ${ }^{18}$ para evitar influências de alterações hormonais e estruturais do envelhecimento, como também as alterações do período da muda vocal ${ }^{17,19-24}$;

Considerou-se como critérios de exclusão os casos de:

- presença de hábitos de etilismo e/ou tabagismo, por esses agentes serem agressivos à laringe ${ }^{25}$;

- ser cantora, instrumentista de sopro ou desportista, pois sabe-se que esses indivíduos podem apresentar alterações de $\mathrm{TMF}^{3,4,14}$;

- ter realizado terapia vocal prévia ou ter conhecimento das técnicas de treinamento respiratório, pois desenvolvem novo condicionamento pneumofônico com as técnicas terapêuticas;

- apresentar qualidade vocal com alteração em grau moderado a intenso, pois subentende alteração glótica que poderia limitar a área glótica mesmo durante a emissão dos sons surdos;

- presença de alterações auditivas à triagem auditiva, pois as mesmas poderiam modificar o automonitoramento da produção da $\mathrm{voz}^{4,5}$;

- presença de alterações do sistema estomatognático que interferissem na articulação dos fonemas-alvo deste estudo;

- alterações evidentes e/ou relatadas durante a entrevista, nos aspectos neurológico, psiquiátrico, cognitivo e ou pulmonar, pois poderiam influenciar a produção vocal;

- relato de antecedentes de doenças respiratórias (asma, tuberculose, bronquite crônica) ou de cirurgia torácica;

- apresentar gripe, crise de alergia respiratória ou outra doença que pudesse interferir no momento das avaliações.

Para a aplicação dos critérios citados, os sujeitos da primeira etapa foram selecionados através da análise de todas as suas avaliações armazenadas (as mesmas dos sujeitos captados em campo), visando aos critérios de inclusão e de exclusão.

Nos sujeitos captados em campo, foram realizadas a entrevista inicial, que contava com a coleta 
da estatura auto-referida, a avaliação miofuncional orofacial, a triagem auditiva, e a análise vocal perceptivo-auditiva por meio da escala GRBAS $^{3}$ para selecionar os sujeitos que se enquadrassem nos critérios de inclusão e de exclusão e definir a amostra.

A triagem auditiva foi realizada por meio de uma varredura de tons puros nas frequências de $500,1000,2000$ e $4000 \mathrm{~Hz}$, à intensidade de $25 \mathrm{~dB}$ NA, somente por via aérea, em sala acusticamente tratada, para identificar eventuais presenças de alterações auditivas; ou considerou-se a apresentação do laudo de avaliação auditiva.

Para a exclusão dos sujeitos com alterações vocais, realizou-se triagem perceptivo-auditiva da voz com as gravações de fala espontânea do banco de dados, e com a gravação da entrevista, na segunda etapa da pesquisa, julgando-se a voz por meio da escala GRBAS ${ }^{3}$. Foram excluídos todos os sujeitos que apresentaram alteração vocal perceptivo-auditiva de grau moderado a intenso, pois uma disfonia nesses graus pode ser indicativa de alteração em pregas vocais que poderia diminuir a área glótica mesmo durante a emissão dos sons surdos utilizados neste estudo ${ }^{3}$.

Após o processo de amostragem, conforme a aplicação dos critérios de inclusão e de exclusão obteve-se 24 registros do Banco de Dados e 24 sujeitos selecionados da coleta em campo, totalizando um grupo de 48 sujeitos que passaram à coleta de dados para a pesquisa.

Para a coleta dos valores de TMF das emissões de /è/ e de /s/, os sujeitos foram orientados a permanecer em posição ortostática bípede. A emissão foi feita de forma sustentada e contínua, emitindo o som surdo em tempo máximo de fonação, com intensidade habitual, sendo as medidas registradas por cronômetro $3,4,8,17,20,22$. Para a emissão do TMF /è/, os indivíduos foram orientados a emitirem o fonema áfono /e/ de forma prolongada sem vocalização, mas mantendo a postura articulatória da emissão da vogal /e/, ou, conforme a autora que propõe essa medida 4 (p.29.) instrui: "com controle consciente para não pressionar a glote, produzindo uma emissão como se fosse um bafinho muito leve para embaçar um espelho". Esse procedimento foi realizado três vezes para cada fonema pesquisado, sendo considerado o maior tempo obtido para cada $u^{3,4,8,17,20,22}$.

O trabalho foi previamente aprovado pelo Comitê de Ética em Pesquisa da Universidade Federal de Santa Maria (número 23081.008439/2007-16). $\mathrm{Na}$ primeira etapa, os sujeitos haviam assinado o Termo de Consentimento Livre e Esclarecido (TCLE) que a clínica-escola oferece e que prevê a possibilidade de utilização dos dados coletados em pesquisas futuras. A segunda etapa iniciou após leitura e assinatura do TCLE específico da pesquisa (res.196/96, CONEP) por todos os sujeitos.

Ao final da coleta de dados, os valores obtidos foram tabulados e submetidos à análise estatística descritiva das variáveis numéricas: CV, estatura, TMF/s/ e TMF/è/ para os 48 sujeitos; com valores de média, desvio-padrão, valores mínimo, máximo e mediana, para caracterizar os resultados da amostra.

O teste de normalidade de Shapiro-Wilk foi aplicado às variáveis para verificar a ocorrência de distribuição Normal; também foi calculado o coeficiente de variação para verificar a homogeneidade das variáveis de TMF. Adotou-se o nível de significância de $5 \%$, ou seja, $\mathrm{P}<0,05$, para os testes estatísticos.

Para análise estatística, foi utilizado o seguinte programa computacional: The SAS System for Windows (Statistical Analysis System), versão 8.02. SAS Institute Inc, 1999-2001, Cary, NC, USA.

\section{RESULTADOS}

Os resultados obtidos estão expostos nas tabelas numeradas de 1 a 3 e no gráfico 1. A tabela 1 mostra a análise descritiva da CV, TMF/s/, TMF/è/ e estatura, apresentando média, mediana, desviopadrão e valores mínimo e máximo dessas quatro variáveis estudadas.

A tabela 2 expõe os resultados do teste de Normalidade Shapiro-Wilk para as variáveis pesquisadas, com seus valores demonstrando que apenas a variável TMF/è/ não apresentou distribuição normal.

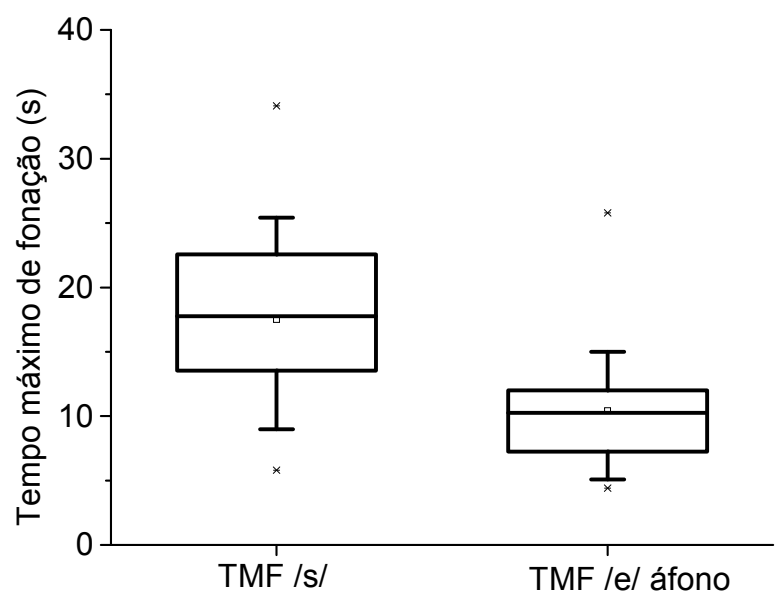

Legenda: TMF/s/=tempo máximo de fonação de /s/; TMF/è/= tempo máximo de fonação de /e/ áfono

Figura 1 - Análise gráfica das variáveis numéricas de tempo máximo de fonação de /s/e tempo máximo de fonação de /e/ áfono 
Tabela 1 - Análise descritiva das variáveis numéricas de capacidade vital, tempo máximo de fonação de /s/, tempo máximo de fonação de lè/ e estatura

\begin{tabular}{ccccccc}
\hline VARIÁVEL & $\mathbf{N}$ & MÉDIA & MEDIANA & D.P. & MÍN & MÁX \\
\hline CV (ml) & 48 & 3206,3 & 3300,0 & 512,98 & 2300,0 & 4400,0 \\
TMF/s/ (s) & 48 & 17,49 & 17,75 & 6,23 & 5,80 & 34,10 \\
TMF/è/ (s) & 48 & 10,43 & 10,25 & 4,08 & 4,40 & 25,80 \\
Estatura (m) & 48 & 1,65 & 1,65 & 0,07 & 1,53 & 1,78 \\
\hline
\end{tabular}

Legenda: $n=$ número de áreas $(n=48) ; D . P .=$ desvio padrão; $C V=$ capacidade vital; TMF/s/=tempo máximo de fonação de /s/; TMF/ è/=tempo máximo de fonação de /e/ áfono; s=segundos; $m=$ =metros.

Tabela 2 - Teste de Normalidade Shapiro-Wilk para as variáveis numéricas de capacidade vital, tempo máximo de fonação de /s/, tempo máximo de fonação de /è/ e estatura

\begin{tabular}{cccc}
\hline VARIÁVEL & W & P & Normalidade \\
\hline CV $(\mathbf{m l})$ & 0,97 & $=0,264$ & não houve desvio significativo da normalidade \\
TMF/s/ $(\mathbf{s})$ & 0,98 & $=0,783$ & não houve desvio significativo da normalidade \\
TMF/è/ $(\mathbf{s})$ & 0,89 & $<0,001$ & houve desvio significativo da normalidade \\
Estatura (m) & 0,97 & $=0,236$ & não houve desvio significativo da normalidade \\
\hline
\end{tabular}

Legenda: $W=$ teste de normalidade Shapiro-Whilk; $P=$ nível de significância; $C V=$ capacidade vital; TMF/s/=tempo máximo de fonação de /s/; TMF/è/=tempo máximo de fonação de /e/ áfono; s=segundos; m=metros.

A tabela 3 apresenta os valores dos Coeficientes de Variação dos TMF de /s/ e /è/, evidenciando valores muito próximos à sua análise de homogeneidade. O gráfico 1 mostra a distribuição de ambos os TMF surdos pesquisados em função do tempo.

Tabela 3 - Coeficiente de Variação para a análise de homogeneidade das variáveis numéricas de tempo máximo de fonação de /s/ e tempo máximo de fonação de lè/

\begin{tabular}{cc}
\hline VARIÁVEL & Coeficiente de Variação \\
\hline TMF/s/ (s) & $35,60 \%$ \\
TMF/è/ (s) & $39,11 \%$ \\
\hline
\end{tabular}

Legenda: TMF/s/=tempo máximo de fonação de /s/; TMF/è/= tempo máximo de fonação de /e/ áfono

\section{DISCUSSÃO}

Os valores de $\mathrm{CV}$ e de $\mathrm{TMF} / \mathrm{s} /$ deste estudo apresentaram distribuição normal (tabela 2), sendo sugeridos como medidas que podem contribuir com a faixa de normalidade para a avaliação fonoaudiológica de mulheres adultas. Por seguir a distribuição normal (curva de Gauss) dentro do grupo estudado, esses resultados poderão ser um ponto de referência para a avaliação fonoaudiológica de mulheres adultas, inclusive porque os valores encontrados são corroborados pela literatura ${ }^{2-8,13-17,26}$. A estatura média do grupo foi de $1,65 \mathrm{~m}$, sendo que as medidas da estatura dos sujeitos da pesquisa também apresentaram um desvio-padrão próximo de zero $(\mathrm{DP}=0,07)$, conferindo uma qualidade homogênea aos padrões de estatura dos indivíduos da amostra analisada.

Os valores de TMF/è/, apesar de não terem apresentado distribuição normal, mostraram valores de média e mediana bastante próximos $(10,43 \mathrm{~s}$ e $10,25 \mathrm{~s}$ ), coeficiente de variação muito próximo do TMF/s/ (tabela 3), sendo também importantes como característica do grupo estudado, uma vez que não foram encontradas pesquisas estudando valores para esta medida, apenas a referência nacional teórica, sem pesquisa de campo, que a propõe ${ }^{4}$ e uma pesquisa de campo que se utilizou da medida ${ }^{8}$ e de seus valores de referência propostos teoricamente $^{4}$. No entanto, acredita-se que não possam ser sugeridos como valores balizadores da normalidade do TMF/è/ devido à distribuição não-normal, que sugere a presença de alguma variável interveniente, e à carência de pesquisas de campo que legitimizem esta medida.

Por se tratarem de medidas amplamente utilizadas na prática clínica, o conhecimento dos valores de TMF que caracterizam grupos 
considerados normais, por meio do cálculo de normalidade, é essencial para interpretar as avaliações objetivas, complementando a análise perceptivo-auditiva da voz, fornecendo mais evidências para a caracterização vocal, diagnóstico diferencial, intervenção terapêutica e acompanhamento da evolução ${ }^{1,3,4,8,15,16}$.

Os valores de CV e TMF variam de acordo com o sexo, raça, faixa etária, altura, condições de saúde, tipo de profissão, e hábitos como fumo, prática de esportes, canto, instrumentos de sopro 1,7,8,10-12,15,26-28, sendo, portanto, importante a identificação e controle de tais variáveis para reduzir as variações nos resultados e na interpretação da avaliação vocal ${ }^{26}$, conforme ocorreu neste estudo através dos critérios de inclusão e de exclusão adotados.

A CV dos sujeitos da amostra (tabela 1) mostrou valores médios e medianos dentro da normalidade referida pela literatura, a qual refere valores de $2.100 \mathrm{ml}$ a $3.300 \mathrm{ml}$ para adultos do sexo feminino. Considera-se que valores inferiores a $2.100 \mathrm{ml}$ são insuficientes para cumprir com eficácia a função fonatória ${ }^{3}$. Medidas reduzidas de CV podem representar dificuldade para sustentar a fonação e tensão laríngea, na tentativa de manter a emissão, além de inspirações frequentes com pausas inadequadas no discurso, e contração da musculatura extrínseca do pescoço $\mathrm{O}^{3,4,8,13}$.

Os TMF representam a duração máxima que o sujeito é capaz de sustentar um som em uma expiração prolongada ${ }^{1-4,6-8,15,16}$. Com valores normais de $\mathrm{CV}$, e sem relato de doenças neurológicas ou respiratórias ${ }^{8}$, os indivíduos desta pesquisa contavam com volume de ar suficiente para sustentar as emissões surdas durante os tempos máximos esperados pois, para a produção da fonação máxima, o indivíduo utiliza sua CV para sustentar a emissão pelo maior tempo possível $\mathrm{j}^{3,4,8,15,29}$.

A análise dos TMF/s/ mostrou que os valores médios e medianos da amostra do estudo (tabela 1) ficaram dentro da normalidade de adultos do sexo feminino, em torno de 15 a 25 segundos $^{3,4,8,13,16}$. Em pesquisa recente ${ }^{15}$, realizou-se a média dos valores de normalidade de TMF das fricativas /s/ e /z/ referenciados em literatura por diversos autores, encontrando-se, como valores de normalidade, o intervalo de 15,57 a 34,17 segundos para o sexo feminino, também compatível com os achados do presente trabalho.

TMF de sons surdos como o /s/ e o /è/ não têm vibração de pregas vocais, dispensando o uso da fonte glótica para sua produção. Eles indicam a habilidade de controle expiratório à fonação, fornecendo dados da dinâmica respiratório-fonatória do indivíduo $0^{3-6,8,16}$. Como a produção da emissão do som de /s/ contínuo não envolve coaptação glótica, o controle da saída do ar provavalmente é realizado pela constrição articulatória ${ }^{4-6,8,16}$.

Para o TMF da vogal áfona /e/ em adultos, a normalidade apontada é de 16 a 18 segundos ${ }^{4,8}$. $\mathrm{Na}$ presente pesquisa, verificou-se que os valores médios e medianos de TMF/è/ da amostra (tabela 1) foram significativamente menores do que os valores da normalidade proposta ${ }^{4}$ teoricamente. De modo geral, os resultados dos TMF estão relacionados com o grau de adução das pregas vocais e com as dificuldades de controle respiratório ${ }^{1-9}$, ou seja, quanto menor o TMF, maior o distúrbio de controle glótico e ou respiratório. Como esta emissão não possui controle glótico e tampouco articulatório à saída do $a^{4}$, a sustentação de seu tempo de emissão depende quase que exclusivamente do controle respiratório.

O grupo estudado foi apenas de mulheres normais no aspecto respiratório/pulmonar, considerando-se os resultados estatísticos com distribuição normal dentro do proposto pela literatura (valores de referência adotados neste trabalho), revelando a homogeneidade da amostra, fator importante para minimizar as demais variáveis. Assim, é possível que em amostras diferentes, por exemplo quanto ao sexo, os resultados pudessem ser mais heterogêneos e talvez se aproximar do proposto pela única referência teórica sobre o TMF/è/4, uma vez que a autora não menciona se a sua faixa de normalidade inclui ambos os sexos, pois, de maneira geral, os TMF femininos são inferiores aos masculinos $3,4,8,10,11,15,27$, devido especialmente ao fato de sujeitos do sexo masculino possuirem estrutura pulmonar maior do que o feminino, resultando em maior CV, da mesma forma que em indivíduos maiores ou de porte atlético ${ }^{3,11,15,26}$.

Como a amostra foi de mulheres adultas, sem alterações que interferissem nas variáveis estudadas, e como os valores de CV e de TMF/s/ apresentaram distribuição normal, dentro do esperado para a literatura ${ }^{3,4,8,13-16}$, e coeficiente de variação muito próximos entre TMF/s/ e TMF/è/, é possível que esses valores reflitam a característica de normalidade de TMF/è/ deste grupo de mulheres adultas normais, mesmo estando abaixo das únicas referências encontradas na literatura ${ }^{4,8}$.

Os resultados estatísticos das medidas de $\mathrm{TMF} / \mathrm{s} /$ e da CV, obtidos neste trabalho, caracterizam o grupo estudado de mulheres adultas consideradas normais do ponto de vista laríngeo e respiratório, concordando com os padrões de normalidade atualmente utilizados para $\mathrm{CV}$ e $\mathrm{TMF} / \mathrm{s} /$ em sujeitos adultos do sexo feminino, ou seja, os resultados dessas medidas ficaram dentro 
do que a literatura considera normal e de referência para o sexo feminino ${ }^{3,4,8,13-16}$.

Paralelamente, os resultados evidenciaram valores diferentes da literatura ${ }^{4,8}$ para o TMF/è/ em mulheres adultas normais, fornecendo indícios de uma possível variação em relação à única referência de normalidade encontrada na literatura ${ }^{4}$, sendo interessantes para futuras pesquisas envolvendo esta importante medida.

Salienta-se que estudos futuros podem envolver ambos os sexos, diferentes estaturas e faixas etárias; além de estender a pesquisa a populações com diferentes tipos de patologias laríngeas e respiratórias, abrangendo, assim, uma ampla zona de investigação dessas medidas de avaliação, levando em consideração seus fatores intervenientes.

\section{CONCLUSÃO}

Para o grupo de mulheres de 18 a 44 anos de idade e estatura média de $1,65 \mathrm{~m}$, analisado neste trabalho, encontraram-se valores médios de CV e de TMF/s/ compatíveis com os referidos pela literatura.

Os valores médios de TMF/è/ se apresentaram abaixo do proposto pela única referência teórica existente, evidenciando a necessidade de mais pesquisas como esta - que investiguem em campo a medida de TMF/è/ - a fim de estabelecer a faixa normalidade conforme o sexo e obter mais dados científicos sobre o que parece ser a medida mais indicada para a avaliação isolada do controle respiratório durante a emissão.

\begin{abstract}
Purpose: to check the vital capacity (VC) and the maximum time values of phonation (MTP) as for the voiceless /e/ (represented by /é/) and the /s/ in adult women, comparing it with the proposed normality pattern. Method: collecting VC, MTP/é/, MTP/s/values and self-reported stature of 48 women between 18 and 44-year old, normal condition according to otorhinolaryngological, myofunctional, vocal, breathing and hearing ratings. We applied descriptive statistical analysis, Shapiro-Wilk normality test and calculated the coefficient variation; we adopted a 5\% significance level. Results: mean values: $3.206 \mathrm{ml}$ as for VC, $17.49 \mathrm{~s}$ as for MTP/s/ and $1.65 \mathrm{~m}$ as for height, with normal distribution, MTP/é/ did not have a normal distribution and whose 10.43 s average was significantly lower than the literature values $(P<0.001)$. However, MTP/é/ showed quite close medium and median values $(10.43$ and $10.25 \mathrm{~s}$ ) and coefficient variation very similar to the MTP/s/. Conclusion: for the analyzed group, we found medium VC and MTP values compatible with those reported in the literature. and medium MTP/é/ values below those proposed in the literature. The average values of MTP/é/ were found to be below the one proposed by the existing theoretical framework, showing the need for more similar studies - to investigate the field of measuring MTP/é/ - in order to set up the normal range according to gender and more scientific data on what appears to be the most suitable for the separate evaluation of respiratory control during emission
\end{abstract}

KEYWORDS: Voice; Vocal Cords; Vital Capacity; Voice Disorders

\section{REFERÊNCIAS}

1. Dehqan, A.; Ansari, H.; Bakhtiar, M. Objective voice analysis of Iranian speakers with normal voices. J Voice, 2010;24(2):161-7.

2. Carrara-de Angelis E. Voz nos distúrbios neurológicos. In: Ferreira LP, Befi-Lopes DM, Limongi SCO, organizadoras. Tratado de fonoaudiologia. São Paulo: Roca; 2005. p. 75-90.

3. Behlau MS, organizadora. Voz: o livro do especialista. vol I. 2. ed. Rio de Janeiro: Revinter; 2008.

4. Pinho SMR. Fundamentos em fonoaudiologia: tratando os distúrbios da voz. 2. ed. Rio de Janeiro: Guanabara Koogan; 2003.
5. Andrews ML. Manual de tratamento da voz: da pediatria à geriatria. 3. ed. São Paulo: Cengage Learning; 2009. 652 p.

6. Cielo CA, Casarin MT. Sons fricativos surdos. Rev CEFAC. 2008;10(3):352-8.

7. Awan SN. The aging female voice: acoustic and respiratory data. Clin Linguist Phon. 2006;20(2/3):171-80.

8. Rossi DC, Munhoz DF, Nogueira CR, Oliveira TCM, Britto ATBO. Relação do pico de fluxo expiratório com o tempo de fonação em pacientes asmáticos. Rev CEFAC. 2006;8(4):509-17.

9. Behlau M, Madazio G, Feijó D, Azevedo R, Gielow I, Rehder MI. Aperfeiçoamento vocal e tratamento fonoaudiológico das disfonias. In: Behlau MS, 
organizadora. Voz: O livro do especialista. vol II. Rio de Janeiro: Revinter; 2005. p. 417-525.

10. Bellia V, Catalano F, Pistelli R, Antonelli-Incalzi R. Aging on quality of spirometry. Am J Respir Crit Care Med. 2000;161(1):1094-100.

11. Behlau MS. Técnicas vocais. In: Ferreira LP, Befi-Lopes DM, Limingi SCO, organizadoras. Tratado de fonoaudiologia. São Paulo: Roca; 2004. p. 42-58.

12. Linville SE. The aging voice. The ASHA Leader. 2004;9(19):12-21.

13. Bortolotti P, Silva MA. Caracterização da voz de um grupo de mulheres com obesidade mórbida acompanhadas no setor de cirurgia bariátrica da Irmandade Santa Casa de Misericórdia de São Paulo. Distúrbios da Comunicação. 2005;17(2):149-60.

14. Rosa JC, Cielo CA, Cechella C. Função fonatória em pacientes com doença de Parkinson: uso de instrumento de sopro. Rev CEFAC. 2009;11(2):305-13.

15. Beber BC, Cielo CA, Siqueira MA. Lesões de borda de pregas vocais e tempos máximos de fonação. Rev CEFAC. 2009;11(1):134-41.

16. Cielo CA, Conterno G, Carvalho CDM, Finger LS. Disfonias: relação $s / z$ e tipos de voz. Rev CEFAC. 2008;10(4):536-47.

17. Finger LS, Cielo CA, Schwarz K. Medidas vocais acústicas de mulheres sem queixas de voz e com laringe normal. Braz $\mathrm{J}$ Otorhinolaryngol. 2009;75(3):432-40.

18. Descritores em Ciências da Saúde. DeCS Biblioteca Virtual em Saúde, 2009 [página online]. Disponível em: <http://decs.bvs.br/cgi-bin/wxis1660. exe/decsserver/>. Acesso em: out. 2009.

19. Felippe ACN, Grillo MHMM, Grechi TH. Normatização de medidas acústicas para vozes normais. Rev Bras Otorrinolaringol. 2006;72(5):659-64.
20. Finger LS, Cielo CA. Aspectos fisiológicos e clínicos da técnica fonoterapêutica de fonação reversa. Rev Bras Otorrinolaringol. 2007;73(2):271-7.

21. Bolzan GP, Cielo CA, Brum DM. Efeitos do som basal em fendas glóticas. Rev CEFAC. 2008;10(2):218-25.

22. Finger LS, Cielo CA. Modificações vocais acústicas produzidas pela fonação reversa. Rev Soc Bras Fonoaudiol. 2009;14(1):15-21.

23. Gama ACC, Behlau MS. Estudo da constância de medidas acústicas de vogais prolongadas e consecutivas em mulheres sem queixa de voz e em mulheres com disfonia. Rev Soc Bras Fonoaudiol. 2009;14(1):8-14.

24. Roman-Niehues G, Cielo CA. Modificações vocais acústicas produzidas pelo som hiperagudo. Rev CEFAC. 2010; 12(3):462-70.

25. Awan SN, Morrow DL. Videostroboscopic characteristics of young adult female smokers vs. nonsmokers. J Voice. 2006;21(2):211-23.

26. Pereira CAC, Sato T, Rodrigues SC. Novos valores de referência para espirometria forçada em brasileiros adultos de raça branca. J Bras Pneumol. 2007;33(4):397-406.

27. Joseph CLM, Ownby DR, Peterson EL, Johnson CC. Racial differences in physiologic parameters related to asthma among middle-class children. Chest. 2000;117(1):1336-44.

28. Chinn S, Jarvis D, Svanes C, Burney $P$. Sources of variation in vital capacity. Eur Respir J. 2006;27(4):767-73.

29. Cerceau JSB, Alves CFT, Gama ACC. Análise acústica da voz de mulheres idosas. Rev CEFAC. 2009;11(1):142-9.
http://dx.doi.org/10.1590/S1516-18462011005000047

RECEBIDO EM: 19/08/2010

ACEITO EM: 16/02/2011

Endereço para correspondência:

Carla Aparecida Cielo

Rua Pedro Londero, 155

Santa Maria - RS

CEP: 97095-530

E-mail: cieloca@yahoo.com.br 